\title{
On the Choice of Filter Bank Parameters for Wavelet-Packet Identification of Dynamic Systems
}

\author{
Henrique Mohallem Paiva and Roberto Kawakami Harrop Galvão \\ Instituto Tecnológico de Aeronáutica - ITA \\ CTA, São José dos Campos, SP, 12228-900, Brazil \\ \{hmpaiva, kawakami\}@ita.br
}

\begin{abstract}
This paper is concerned with a recently proposed technique for linear system identification in frequency subbands using waveletpacket filter banks. More specifically, the effect of using different mother wavelets and resolution levels is investigated. The study is based on simulated examples involving the identification of a servomechanism model. The results reveal that the identification outcome can be improved by using wavelet filters with better frequency selectivity, as well as by increasing the number of resolution levels in the filter bank. In this context, the advantages of using wavelet packets instead of standard wavelet decompositions are also discussed.
\end{abstract}

Keywords: Wavelet Packets, System Identification, Filter Banks.

\section{Introduction}

System identification techniques employing wavelet decompositions have been widely used in several applications. Examples include, for instance, modal parameter estimation in mechanical systems [79], vibration signal modelling [5], and non-parametric linear system identification [11. The use of wavelet packets, which allow greater flexibility in spectral partioning as compared to standard wavelet transforms, has also been proposed in this context [12].

In 12, the wavelet-packet decomposition tree was used to establish frequency bands where subband models were created. The tree structure was optimized by using a generalized cross-validation method in order to achieve a compromise between accuracy and parsimony of the overall model. In this manner, the most appropriate frequency partitioning for the subband models was automatically determined. In comparison with a standard ARX (autoregressive with exogenous output) identification method, the proposed technique was superior in terms of resonance peak identification and sensitivity to white measurement noise. This technique was also studied in [13, where the effects of coloured noise were analyzed. However, some important aspects of concern for system identification were not addressed. In particular, the advantage of using wavelet packets instead of the more conventional wavelet transform was not clearly demonstrated.

A. Elmoataz et al. (Eds.): ICISP 2010, LNCS 6134, pp. 272 279, 2010.

(C) Springer-Verlag Berlin Heidelberg 2010 
Moreover, the choice of mother wavelet and number of resolution levels was not discussed. In fact, choosing the mother wavelet is an important issue in any given application 2, such as texture analysis [1, eletrocardiogram signal processing [4] and denoising [14.

The present work investigates the effect of the choice of wavelet type (family and length) and number of resolution levels in the wavelet-packet identification algorithm proposed in [12]. For this purpose, a case study involving a servomechanism model is presented. The investigation is aimed at providing practical guidelines for using the wavelet packet identification technique. Moreover, a comparison with an identification structure employing the conventional wavelet transform is carried out. In this case, the purpose consists of determining whether the wavelet packet formulation does bring advantages over the use of simpler wavelet decompositions.

\subsection{Notation}

Fig. 1]depicts the wavelet-packet decomposition and reconstruction trees [15]. In this figure, $H$ and $G$ are lowpass and highpass filters, respectively, and $H_{r}$ and $\mathrm{G}_{\mathrm{r}}$ are the associated reconstruction filters. In the present work, these filters are adopted such that the filter bank is orthonormal. $\downarrow 2$ and $\uparrow 2$ denote downsampling and upsampling operations, respectively. $\mathbf{y}$ is the signal to be decomposed. $\mathbf{x}_{i, j}\{\mathrm{y}\}$ represents the wavelet coefficients of signal $\mathbf{y}$ at node $(i, j)$ of the tree, where $j \geq 0$ indicates the resolution level and $i$ is a sequential index ranging from 0 to $2^{j}-1$. Node $(i, j)$ is the parent of nodes $(2 i, j+1)$ and $(2 i+1, j+1)$, which are the children of node $(i, j)$. All nodes without children are called leaf nodes. The depth of the tree is defined as the highest level in which nodes are present. A tree is said to be complete if all leaf nodes are in the same resolution

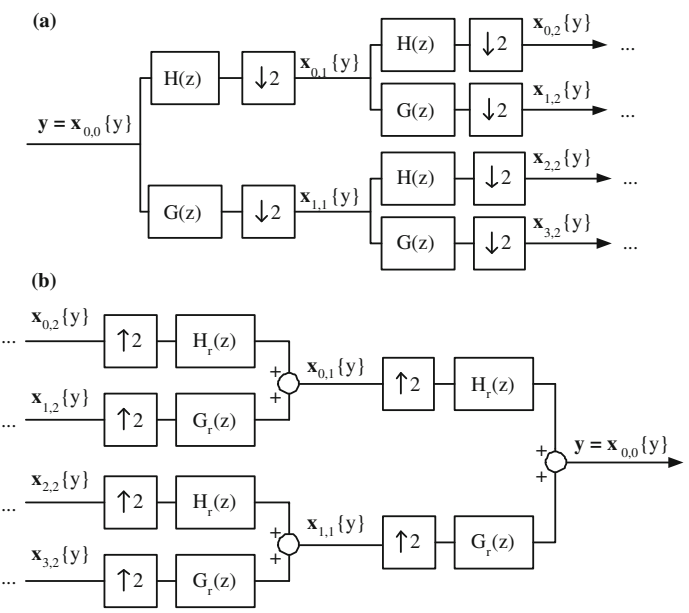

Fig. 1. Wavelet-packet (a) decomposition and (b) reconstruction tree 
level. Scalars and row vectors are represented by italic and boldface lowercase symbols, respectively.

\section{The Wavelet-Packet Identification Technique}

This section presents a short review of the wavelet-packet identification technique proposed in 12. The identification algorithm is based on the development of several subband models. The wavelet-packet decomposition tree (Fig. 1a) is used to establish the frequency bands at which the subband models will be created. Each leaf node of the tree is associated to a frequency band, and the complete set of leaf nodes composes the whole frequency range. For each frequency band, a subband model is created.

This modelling scheme is illustrated in Fig. 2 for a particular structure of the wavelet-packet decomposition tree. In this figure, $\mathrm{M}_{i, j}$ indicates the subband model intended to represent the plant in the frequency band associated to the leaf node $(i, j)$. If $(i, j)$ is a leaf node, then signal $\breve{\mathbf{u}}_{i, j}$ is defined as the output of model $\mathrm{M}_{i, j}$ for input $\mathbf{u}$. If $(i, j)$ is not a leaf node, then the coefficients $\mathbf{x}_{i, j}\left\{\breve{\mathbf{u}}_{i, j}\right\}$ are defined as the reconstruction of the coefficients at the children nodes of $(i, j)$.

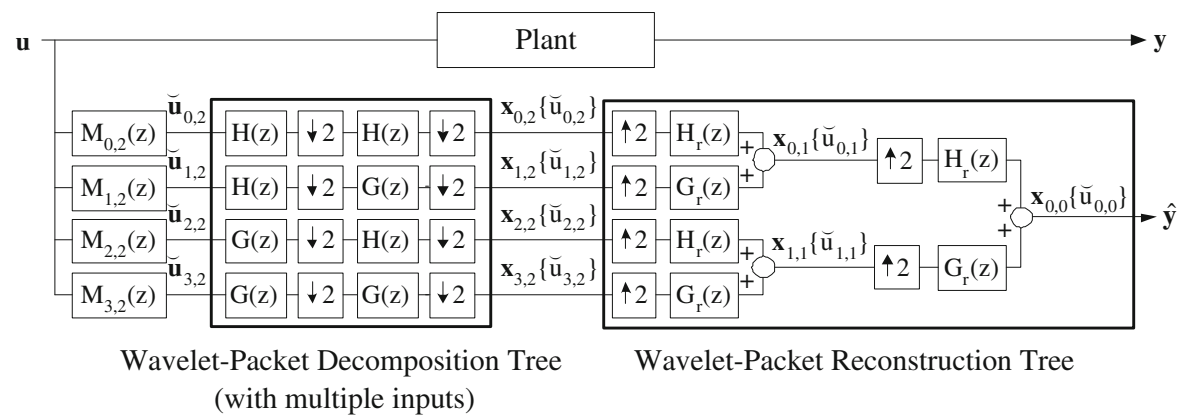

Fig. 2. Example of the system modelling scheme for a particular wavelet-packet decomposition tree with four leaf nodes (in this example, all leaf nodes are in the same level, but this is not a requirement)

The structure adopted for each subband model is a transfer function of the form $\mathrm{M}_{i, j}(\mathrm{z})=\mathrm{P}_{i, j}(\mathrm{z}) \mathrm{Q}_{i, j}(\mathrm{z})$, where

$$
\mathrm{P}_{i, j}(\mathrm{z})=\left[1 /\left(1-\mathrm{z}^{-1}\right)\right]^{s_{i, j}}, s_{i, j} \in \mathbb{Z} ; \quad \mathrm{Q}_{i, j}(\mathrm{z})=\alpha_{i, j}+\beta_{i, j} \mathrm{z}^{-1}, \alpha_{i, j}, \beta_{i, j} \in \mathbb{R}
$$

Parameters $s_{i, j}, \alpha_{i, j}$ and $\beta_{i, j}$ are estimated in order to minimize the following cost function $J_{i, j}: \mathbb{Z} \times \mathbb{R}^{2} \rightarrow \mathbb{R}: J_{i, j}\left(s_{i, j}, \alpha_{i, j}, \beta_{i, j}\right)=\mathbf{e}_{i, j}\left(\mathbf{e}_{i, j}\right)^{\mathrm{T}}$, where residue $\mathbf{e}_{i, j}=\mathbf{x}_{i, j}\left\{\mathrm{y}-\breve{\mathrm{u}}_{i, j}\right\}$ denotes the wavelet-packet coefficients of the difference between the plant output $\mathbf{y}$ and the subband model output $\breve{\mathbf{u}}_{i, j}$, in the frequency band under consideration.

For a fixed value of $s_{i, j}$, cost $J_{i, j}$ is minimized with respect to $\alpha_{i, j}$ and $\beta_{i, j}$ by a least-squares procedure. A search algorithm is used to find the value of 
$s_{i, j}$ that leads to the minimum value of $J_{i, j}$. The details of these procedures are described in [12].

As demonstrated in $\left[12\right.$, the cost $J_{0,0}$, at the root node $(i, j)=(0,0)$ of the wavelet-packet tree, is equal to the square of the 2-norm of the prediction error $(\mathbf{y}-\hat{\mathbf{y}})$, that is, $J_{0,0}=(\mathbf{y}-\hat{\mathbf{y}})(\mathbf{y}-\hat{\mathbf{y}})^{\mathrm{T}}$. Furthermore, for orthonormal wavelets, the cost at a non-leaf node $(i, j)$ was shown to be equal to the sum of the costs at its children nodes, i.e., $J_{i, j}=J_{2 i, j+1}+J_{2 i+1, j+1}$. Such features allow the use of the following algorithm to adjust the wavelet-packet tree structure in order to achieve a compromise between accuracy and complexity of the model [12].

1. Fix the maximum depth $d$ allowed for the tree and initialize the search with a complete tree with that depth.

2. All nodes are candidates to be leaf nodes. Thus, for each node $(i, j)$, leaf or non-leaf, obtain the associated subband model $\mathrm{M}_{i, j}(\mathrm{z})$. Calculate, for each node, the square of the 2 -norm of the residue $\mathbf{e}_{i, j}$ and call this value $J_{i, j}^{l}$. Superscript $l$, standing for leaf, is used to emphasize that $J_{i, j}^{l}$ will be equal to the cost $J_{i, j}$ at node $(i, j)$ only if this node is chosen to be a leaf.

3. Initialize the costs at level $d$, the deepest level of the tree. If one node of this level is kept in the tree, it will be necessarily a leaf node. Thus, let $J_{i, d}=J_{i, d}^{l}$, for all $i=0,1, \ldots,\left(2^{d}-1\right)$.

4. Analyze the other nodes of the tree, which can be made either leaf or nonleaf nodes. Start from level $j=d-1$ and use a bottom-up approach (that is, analyze all nodes of a level before evaluating the parent level). Decide if each node $(i, j)$ should be a leaf or a non-leaf node, by comparing cost $J_{i, j}^{l}$ (cost if $(i, j)$ is a leaf node) with the sum $J_{2 i, j+1}+J_{2 i+1, j+1}$ of the costs at its children nodes (cost if $(i, j)$ is a non-leaf node). The decision rule is:

$$
J_{i, j}=\left\{\begin{array}{l}
J_{i, j}^{l}, \text { if } J_{i, j}^{l} \leq \rho\left(J_{2 i, j+1}+J_{2 i+1, j+1}\right) \\
J_{2 i, j+1}+J_{2 i+1, j+1}, \text { otherwise }
\end{array}\right.
$$

where the penalty factor $\rho \geq 1$ ensures that node $(i, j)$ will only be split into children nodes if the cost reduction is large enough to justify the increase in model complexity.

The penalty factor $\rho$ is required to avoid an overfitting of the identification data. An increase in the value of $\rho$ tends to reduce the number of nodes in the resulting tree. Thus, the choice of the penalty factor $\rho$ can be regarded as a model order determination problem, which is addressed by using a generalized cross validation (GCV) method, described in detail in [12]. The value of $\rho$ that minimizes the GCV index is selected, thus providing a tradeoff between model parsimony and identification accuracy. Therefore, the ability of the model to represent the behaviour of the system for input signals different from the one used in the identification is improved.

\section{Case Study}

This case study involves the identification of a servomechanism model. Eq. (2) represents the transfer function employed in the simulations, where $U$ is the input voltage (volts) and $Y$ is the shaft speed ( $\mathrm{rad} / \mathrm{s})$ of the servomechanism. 


$$
\frac{Y(\mathrm{~s})}{U(\mathrm{~s})}=\frac{5000}{\mathrm{~s}^{3}+75 \mathrm{~s}^{2}+1350 \mathrm{~s}+15000}
$$

The following chirp excitation was used for identification:

$$
u(k T)=\sin (2 \pi f k T), k=0,1, \ldots, 5 \times 10^{4}, T=0.0125 \mathrm{~s}
$$

with frequency $f$ varying linearly from 0.1 to $40 \mathrm{~Hz}$.

A zero-mean, white gaussian noise was added to the output $\mathbf{y}$ of the plant, with standard deviation equal to $5 \%$ of the standard deviation of $\mathbf{y}$.

Sections 3.1 and 3.2 present investigations concerning the choice of the number of resolution levels and the choice of the wavelet filters, respectively.

\subsection{Resolution Levels}

This section presents the results of the identification obtained by using different resolution levels. The Daubechies $8(d b 8)$ wavelet filters were adopted, as in [12]. The maximum wavelet tree depths were varied from 4 to 8 . For comparison, an identification using standard wavelet trees, where only the lowpass channel is successively decomposed, was also performed.

Figure 3 presents the cost $J_{0,0}$ obtained for the standard-wavelet and waveletpacket decomposition trees. As mentioned previously, this cost is equal to the squared 2-norm of the prediction error. The value of $J_{0,0}$ presented in this table is normalized by $\mathbf{y y}^{\mathrm{T}}$ (squared 2-norm of output $\mathbf{y}$ ). For comparison, the squared 2 -norm of the gaussian noise added to the plant output $\mathbf{y}$ is also shown. This value is also normalized by $\mathbf{y y}^{\mathrm{T}}$.

If the identification was perfect, cost $J_{0,0}$ would be equal to the squared 2norm of the noise added to output $\mathbf{y}$. Since the identified model is only an approximation of the true plant, cost $J_{0,0}$ is higher than this value. It is worth noting that $J_{0,0}$ decreases as more resolution levels are used, pointing to an improvement in the approximation. In the wavelet-packet case, $J_{0,0}$ appears to converge to the squared 2-norm of the noise.

It is worth noting that an hypothetic cost $J_{0,0}$ smaller than the squared 2norm of the noise added to output $\mathbf{y}$ would imply data overfitting, i.e., modelling of noise rather than plant dynamics.

\subsection{Wavelet Filters}

This section presents the identification results obtained by using different wavelets. The wavelet tree depth was set to six and the wavelet filters were taken from the Daubechies, Symlet and Coiflet families [6]. It should be noted that the identification technique requires the filter bank to be orthonormal, which means that families such as Morlet, Meyer and Derivative of a Gaussian (DOG) 6] cannot be used.

The wavelet filters $\mathrm{H}, \mathrm{H}_{\mathrm{r}}, \mathrm{G}$, and $\mathrm{G}_{\mathrm{r}}$ (as defined in Fig. 1) corresponding to the $d b N$ and $\operatorname{sym} N$ (Daubechies and Symlet families) have length $2 N$ (each filter 
(a)

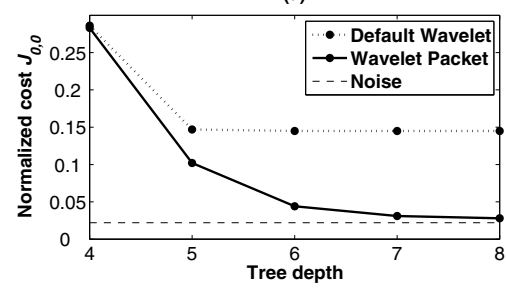

(b)

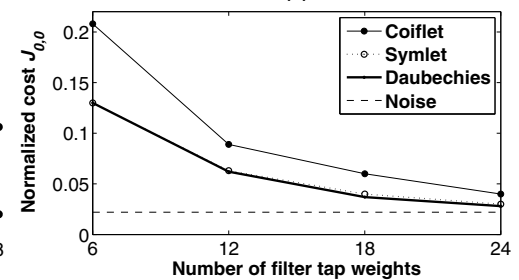

Fig. 3. Servomechanism identification results: Normalized cost $J_{0,0}$ for (a) $d b 8$ wavelet, different resolution levels and (b) different wavelets, six resolution levels
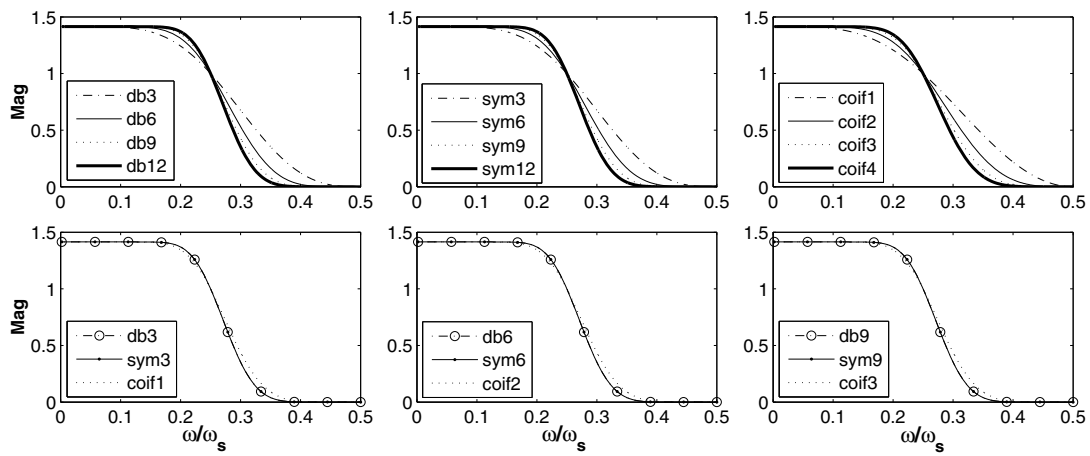

Fig. 4. Frequency response of the low-pass filters $H$ corresponding to mother wavelets from the Daubechies, Symlet and Coiflet families. $\omega_{s}$ denotes the sampling frequency.

has a finite impulse response with $2 N$ values different from zero). The wavelet filters corresponding to the Coiflet family coif $N$ have length $6 N$. Fig. 4 presents the frequency responses of the low-pass filters $\mathrm{H}$ corresponding to the mother wavelets compared in this study. Fig. 3b shows the resulting cost $J_{0,0}$.

Fig. 4 shows that, in a given family, longer wavelets filters present better frequency selectivity (sharper cut-off transition). Furthermore, for a given length, the Daubechies and Symlet filters have very similar frequency responses, with selectivity better than the Coiflet filter.

The results presented in Fig. 3b show that wavelet filters with better frequency selectivity lead to better results (i.e. smaller cost values). The figure shows that Daubechies and Symlet filters with the same number of tap weights lead to similar costs, which are smaller than those obtained with the Coiflet family. Furthermore, in the same family, longer wavelet filters lead to smaller costs. This result was expected because filters with better frequency selectivity lead to a better separation of the frequency bands in the wavelet-packet frequency partitioning scheme.

A comparison between Fig. 3a and Fig. Bb suggests that a reduction in filter length can be compensated by an increase in the wavelet-tree depth, and viceversa. For instance, $d b 12$ with six resolution levels and $d b 8$ with eight resolution 
levels lead to the same cost value (0.028). In terms of computational effort, the most efficient approach would be to use longer filters and less resolution levels (because increasing the filter length increases the complexity linearly, whereas increasing the number of resolution levels may increase the number of leaf nodes exponentially). However, at least a minimum number of resolution levels should be used, in order to maintain the frequency-partitioning flexibility.

\section{Conclusions}

This paper revisited a recently proposed technique for linear system identification in frequency subbands. In the proposed formulation, a wavelet-packet decomposition tree is used to establish frequency bands where subband models are created. An optimization of the tree structure is performed by using a generalized cross-validation method in order to achieve a compromise between accuracy and parsimony of the overall model.

The following conclusions were obtained in the application example involving the identification of a simulated servomechanism:

- Number of resolution levels: The cost associated to the identification was shown to be higher than the squared 2-norm of the noise added to the plant output, and to converge to this value when more resolution levels are used, which indicates an improvement in the identification. The use of wavelet packets led to better results (lower identification costs) than the use of the standard wavelet decomposition. An hypothetic cost lower than the squared 2-norm of the noise, which would imply modelling of noise rather than plant dynamics, was not observed.

- Different wavelets: Wavelet filters with better frequency selectivity provided better cost results. Moreover, the results suggest that a reduction in filter length can be compensated by an increase in the wavelet-tree depth, and vice-versa. In terms of computational effort, the most efficient approach is to use longer filters and less resolution levels; however, at least a minimum number of resolution levels should be used, in order to keep the frequency-partitioning flexibility.

Future works could adopt other basic structures for each subband model $\mathrm{M}_{i, j}$. For instance, more coefficients may be used in the $\mathrm{Q}_{i, j}(\mathrm{z})$ term in Equation (1). Moreover, the use of autoregressive terms could also be investigated.

The model order determination problem, addressed in this work by using the GCV method, could be studied by using alternative approaches, such as model complexity penalty, statistical hypothesis tests [10] and the minimum description length criterion [8]. Finally, it would be worth extending the technique to the identification of a broader class of systems, such as piecewise-affine plants [3].

\section{Acknowledgements}

This work was supported by Embraer, CNPq (research fellowship and postdoctoral grant 200721/2006-2) and FAPESP (grant 2006/58850-6). 


\section{References}

1. Abhayaratne, G.C.K., Jermyn, I.H., Zerubia, J.: Texture-adaptive mother wavelet selection for texture analysis. In: Proc. IEEE International Conference on Image Processing (ICIP), vol. 2, pp. 1290-1293 (2005)

2. Ahuja, N., Lertrattanapanich, S., Bose, N.K.: Properties determining choice of mother wavelet. IEE Proceedings - Visual, Image and Signal Processing 152(5), 659-664 (2005)

3. Bemporad, A., Garrulli, A., Paoletti, S., Vicino, A.: A bounded-error approach to piecewise affine system identification. IEEE Transactions on Automatic Control 50(10), 1567-1580 (2005)

4. Bhatia, P., Boudy, J., Andreao, R.V.: Wavelet transformation and pre-selection of mother wavelets for ECG signal processing. In: Proc. 24th IASTED international conference on Biomedical engineering, pp. 390-395 (2006)

5. Chen, H.X., Chua, P.S.K., Lim, G.H.: Adaptive wavelet transform for vibration signal modelling and application in fault diagnosis of water hydraulic motor. Mechanical Systems and Signal Processing 20(8), 2022-2045 (2006)

6. Daubechies, I.: Ten Lectures on Wavelets. CBMS-NSF Series in Applied Mathematics, vol. (61). SIAM, Philadelphia (1992)

7. Erlicher, S., Argoul, P.: Modal identification of linear non-proportionally damped systems by wavelet transform. Mechanical Systems and Signal Processing 21(3), 1386-1421 (2007)

8. Feil, B., Abonyi, J., Szeifert, F.: Model order selection of nonlinear input-output models: a clustering based approach. Journal of Process Control 14(6), 593-602 (2004)

9. Huang, C.S., Su, W.C.: Identification of modal parameters of a time invariant linear system by continuous wavelet transformation. Mechanical Systems and Signal Processing 21(4), 1642-1664 (2007)

10. Ljung, L.: System Identification: Theory for the User, 2nd edn. Prentice Hall, Upper Saddle River (1999)

11. Luk, R.W.-P., Damper, R.I.: Non-parametric linear time-invariant system identification by discrete wavelet transforms. Digital Signal Processing 16(3), 303-319 (2006)

12. Paiva, H.M., Galvão, R.K.H.: Wavelet-packet identification of dynamic systems in frequency subbands. Signal Processing 86(8), 2001-2008 (2006)

13. Paiva, H.M., Galvão, R.K.H.: Wavelet-Packet Identification of Dynamic Systems with Coloured Measurement Noise. In: Elmoataz, A., Lezoray, O., Nouboud, F., Mammass, D. (eds.) ICISP 2008. LNCS, vol. 5099, pp. 508-515. Springer, Heidelberg (2008)

14. Singh, B.N., Tiwari, A.K.: Optimal selection of wavelet basis function applied to ECG signal denoising. Digital Signal Processing 16(3), 275-287 (2006)

15. Vetterli, M., Kovacevic, J.: Wavelets and Subband Coding. Prentice Hall, Upper Saddle River (1995) 